\title{
Perfil hematólogico e características das fezes de equinos consumindo dietas hiperlipidêmicas
}

\author{
Blood profile and characteristics of the feces of horses fed with high fat diets
}

\author{
Fernanda Nascimento Godoi ${ }^{I}$ Fernando Queiroz AlmeidaII Giuliano Aragonez Guarienti"II \\ Juliano Martins Santiago ${ }^{\text {IV }}$ Daniel Guedes Júnior ${ }^{\text {IV }}$ Yzabella Campos Nogueira $^{\text {IV }}$ \\ Lucas Santiago Brasileiro ${ }^{\mathrm{IV}}$
}

RESUMO

Objetivou-se avaliar os parâmetros sanguíneos, o consumo de matéria seca e as características fecais de equinos alimentados com dietas hiperlipidêmicas. Foram utilizados 15 equinos em delineamento experimental inteiramente casualizado, com três tratamentos e cinco repetições. As dietas utilizadas foram: dieta sem inclusão de óleo de soja (controle); dieta com inclusão de $8,5 \%$ de óleo de soja; dieta com inclusão de $19,5 \%$ de óleo de soja. O ensaio teve duração de 34 dias, sendo 30 dias de adaptação dos equinos às dietas e quatro dias de coleta de amostras. As amostras sanguíneas para avaliação hematológica e bioquímica foram coletadas no 34음 dia, as características das fezes foram avaliadas no $33^{\circ}$ e no $34^{\circ}$ dia do ensaio e, a partir do $30^{\circ}$ dia, o consumo de matéria seca (CMS) foi avaliado. Os equinos alimentados com as dietas hiperlipidêmicas apresentaram aumento no número de eritrócitos e redução no volume corpuscular médio $(P<0,05)$. $O$ nível de hemoglobina dos equinos avaliados diferiu $(P<0,05)$ somente entre os animais que consumiram as dietas de controle e com inclusão de $8,5 \%$ de óleo de soja, com menor valor na dieta de controle de $9,5 d d L^{-1}$. A inclusão de $19,5 \%$ de óleo de soja na dieta elevou os níveis séricos de triglicerídios nos equinos. Houve redução no CMS diário de 1,9 e 2,8kg, nos equinos alimentados com as dietas com 8,5 e 19,5\% de óleo de soja em relação à dieta de controle de $9,0 \mathrm{~kg} \mathrm{MS}$. A produção fecal diária reduziu em 0,7 e $1,1 \mathrm{~kg} \mathrm{MS}$, nos equinos alimentados com as dietas com 8,5 e 19,5\% de óleo de soja, respectivamente, em relação à dieta de controle de $3,4 \mathrm{~kg} \mathrm{MS}$. A utilização de dietas hiperlipidemicas para equinos com inclusão de óleo de soja possibilita a redução do consumo dietético e o aumento da disponibilidade plasmática de triglicerídeos sem a ocorrência de diarreias ou alteração das características das fezes.

Palavras-chave: cavalos, hematologia, lipídios.

\begin{abstract}
This research aimed to evaluate blood parameters, dry matter intake and faeces characteristics in horses fed with high fat diets. Fifteen horses were used in a completely randomized design with three diets and five repetitions. The diets were: diet without soybean oil inclusion (control); diet with of $8.5 \%$ soybean oil inclusion; diet with $19.5 \%$ soybean oil inclusion. The trial had 34 days of duration, 30 days to diet adaptation and four days for samples collection. Blood samples were taken at $34^{\text {th }}$ day to hematological and biochemical evaluation. Faeces characteristics were evaluated from $33^{\text {rd }}$ and $34^{\text {th }}$ day and dry matter intake (DMI) were evaluated from $30^{\text {th }}$ to $34^{\text {th }}$ of essay. Horses fed with high fat diet increased number of erythrocytes and reduced mean corpuscular volume in blood $(P<0.05)$. Blood levels of hemoglobin differ $(P<0.05)$ only in horses fed with control diet or diet with $8.8 \%$ soybean oil inclusion, with lower value in control diet of $9.5 \mathrm{~g} \mathrm{dL}^{-1}$. Serum triglycerides were more available in horses fed diet with $19.5 \%$ soybean oil inclusion. Reduced DMI, of 1.9 and $2.8 \mathrm{~kg}$, were observed in horses fed diet with $8.5 \%$ and $19.5 \%$ soybean oil inclusion respectively in relation to control diet, with DMI of $9.0 \mathrm{~kg} /$ day. Fecal production were reduced, to 0.7 and $1.1 \mathrm{~kg}$ $D M$, in horses fed diets with $8.5 \%$ and $19.5 \%$ soybean oil inclusion in relation to control diet, of $3.4 \mathrm{~kg} D M$. High fat diets for horses with soybean oil inclusion reduced dietetic intake and increased serum triglycerides levels without diarrheas or faeces characteristics alterations.
\end{abstract}

Key words: hematology, horses, lipids.

\section{INTRODUÇÃO}

Atualmente, as provas equestres estão cada vez mais rápidas, com maior grau de dificuldade e

\footnotetext{
IUniversidade Federal de Minas Gerais (UFMG), Belo Horizonte, MG, Brasil.

IIInstituto de Veterinária, Universidade Federal Rural do Rio de Janeiro (UFRRJ), 23890-971, Seropédica, RJ, Brasil. E-mail: falmeida@ufrrj.br. Autor para correspondência.

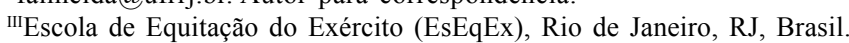

${ }^{\mathrm{IV}}$ Instituto de Veterinária, UFRRJ, Seropédica, RJ, Brasil.
} 
competitividade, exigindo melhor treinamento físico dos equinos e alimentação com maiores teores de energia dietética para suprir as demandas nutricionais. Assim, a inclusão de lipídios nas dietas de equinos atletas é comum, pois esses ingredientes aumentam a concentração energética e reduzem o consumo de grãos, o que diminui a sobrecarga gastrointestinal.

Os óleos vegetais são fontes de energia disponíveis e que podem ser utilizados nas dietas de equinos atletas (HODGSON \& ROSE, 1994), reduzindo os riscos da ocorrência de casos clínicos de cólicas, diarreias e laminites (FRAPE, 2004). A utilização prática de dietas hiperlipidêmicas aumentou o número de pesquisas com o objetivo de avaliar as implicações nos parâmetros hematológicos e bioquímicos dos equinos. Porém, os resultados são contraditórios, o que explica as diferenças quanti e qualitativas dos lipídios fornecidos aos animais (NRC, 2007).

As características das fezes são indicativas do funcionamento do trato gastrointestinal e há poucas informações na literatura relacionando as características das fezes com o manejo alimentar dos equinos. MEYER (1995) relatou que a defecação regular, a consistência, a forma e o cheiro das fezes são critérios importantes para a avaliação do funcionamento do trato digestivo dos equinos, não podendo ser ignorados na avaliação do bem-estar dos equinos e nas alterações dietéticas. Segundo GONÇALVES et al. (2006), avaliando as características das fezes dos equinos, é possível identificar a ocorrência de diarreias e compactações e auxiliar no diagnóstico da síndrome cólica.

Neste estudo, objetivou-se avaliar os parâmetros hematológicos e bioquímicos sanguíneos, o consumo de matéria seca e as características das fezes de equinos consumindo dietas com diferentes níveis de inclusão de óleo de soja.

\section{MATERIAL E MÉTODOS}

O ensaio foi conduzido na Escola de Equitação do Exército, e as análises laboratoriais foram realizadas no Laboratório de Pesquisas em Saúde Equina do Instituto de Veterinária e no Laboratório de Bromatologia Animal do Instituto de Zootecnia da Universidade Federal Rural do Rio de Janeiro. Foram utilizados 15 equinos das modalidades de Adestramento e Salto, mestiços, com média de idade de 7,6 $6 \pm 3,5$ anos e peso médio de $457 \pm 31 \mathrm{~kg}$. Foi utilizado o delineamento experimental inteiramente casualizado com três tratamentos (dietas) e cinco repetições (animais). $\mathrm{O}$ ensaio experimental teve duração de 34 dias, sendo 30 dias de adaptação dos equinos às dietas e quatro dias de coleta de amostras.
As dietas foram definidas como: dieta sem inclusão de óleo de soja (controle) composta por concentrado comerciala $^{\text {a }}$ farelo de soja, sal comum e feno de coastcross (Cynodon dactylon (L) Pers); dieta com inclusão de $8,5 \%$ de óleo de soja composta por concentrado comerciala ${ }^{a}$, farelo de soja, óleo de soja, mistura mineral, sal comum e feno de coastcross; dieta com inclusão de $19,5 \%$ de óleo de soja composta por concentrado comercial ${ }^{\text {a }}$, farelo de soja, óleo de soja, mistura mineral, sal comum e feno de coastcross. As dietas foram formuladas segundo as exigências nutricionais de equinos adultos, a atividade física e o peso dos animais (NRC, 1989). A análise bromatológica dos alimentos foi realizada segundo procedimentos descritos por SILVA \& QUEIROZ (2002), e a energia digestível dos ingredientes foi estimada segundo ALMEIDA et al. (1999), com exceção da fonte lipídica que foi considerada 9,0 Mcal kg-1 de óleo de soja (FRAPE, 2004) (Tabela 1).

O óleo de soja foi incluído nas dietas dos equinos de forma gradual, sendo utilizadas, no mínimo, quatro etapas de adaptação dos animais às dietas hiperlipidêmicas até alcançar 8,5 e 19,5\% de inclusão de óleo de soja. O período de adaptação em cada etapa foi de, no mínimo, cinco dias, e a última teve duração de sete dias, antes da coleta das amostras.

O concentrado comercial ${ }^{a}$ foi gradualmente retirado das dietas hiperlipidêmicas para mantê-las isoenergéticas, sendo necessária a redução na quantidade de feno fornecida aos equinos visando a manter em todas as dietas a relação de concentrado: volumoso de 67:33, na base da matéria seca. O farelo de soja foi acrescentado às dietas para manter a relação de $40 \mathrm{~g}$ de proteína bruta para cada Mcal de energia digestível (NRC, 1989). O fornecimento diário das dietas foi fracionado em cinco vezes, sendo a ração

Tabela 1 - Composição nutricional das dietas experimentais dos equinos (base MS).

\begin{tabular}{llcc}
\hline & \multicolumn{3}{c}{ Níveis de inclusão de óleo } \\
Item & \multicolumn{3}{c}{ de soja, \% } \\
& 0 & 8,5 & 19,5 \\
\hline Matéria Seca (\%) & 90,48 & 91,17 & 92,05 \\
Matéria Orgânica (\%) & 89,46 & 90,42 & 91,83 \\
Proteína Bruta (\%) & 10,51 & 13,05 & 15,72 \\
Extrato Etéreo (\%) & 4,95 & 12,20 & 21,75 \\
Fibra em Detergente Neutro (\%) & 53,83 & 47,34 & 39,46 \\
Fibra em Detergente Ácido (\%) & 22,75 & 21,17 & 19,14 \\
Celulose (\%) & 14,19 & 13,58 & 12,74 \\
Hemiceluloses (\%) & 30,64 & 25,80 & 20,10 \\
Energia Digestível (Mcal dia ${ }^{-1}$ ) & 23,62 & 23,20 & 23,30 \\
\hline
\end{tabular}


concentrada e o óleo de soja fornecidos, em frações iguais, três vezes ao dia, às 4,13 e $20 \mathrm{~h}$, e o feno de coastcross fornecido também em frações iguais, duas vezes ao dia, às 11 e 16h, seguindo o manejo alimentar da Unidade Militar.

Os animais foram exercitados diariamente em atividade física moderada e, a partir do $30^{\circ}$ dia, os equinos foram exercitados ao passo, uma vez ao dia, durante 10 minutos. As amostras de sangue foram coletadas no $34^{\circ}$ dia com os equinos em repouso, às $13 \mathrm{~h}$, imediatamente antes do fornecimento da ração concentrada (KINGSTON, 2008).

Para avaliação hematológica, as amostras de sangue foram coletadas em tubos a vácuo contendo EDTA e refrigeradas a $4^{\circ} \mathrm{C}$. Foram realizadas as análises do volume globular (VG) pelo método de microhematócrito. A hematimetria e a leucometria global foram avaliadas por meio da contagem de células em câmara de Neubauer, e a leucometria específica foi avaliada por meio da avaliação de esfregaço sanguíneo corado pelo kit Panótico rápido em microscopia óptica (COLES, 1984). A concentração de sólidos totais e do fibrinogênio foi realizada por meio de refratometria e, da hemoglobina, por espectrofotometria. Os índices hematimétricos volume corpuscular médio (VCM) e concentração de hemoglobina corpuscular média (CHCM) foram obtidos por meio da relação de parâmetros acima descritos (SMITH, 1993).

Para avaliação da bioquímica sanguínea, as amostras de sangue foram coletadas em tubos a vácuo contendo fluoreto de sódio para determinação de glicose e em tubos a vácuo sem anticoagulante para determinação de triglicerídios, colesterol, ureia, creatinina, $\gamma$-glutamil transferase (GGT), creatinaquinase $(\mathrm{CK})$ e aspartato aminotransferase (AST). Após a coleta, as amostras foram centrifugadas a 7.000rpm, por 10 minutos, para separação do plasma e soro. Foram retiradas alíquotas de $1 \mathrm{~mL}$ com pipeta automática e armazenadas a $-20^{\circ} \mathrm{C}$, em tubos de polipropileno de $1,5 \mathrm{~mL}$, até o momento da análise. As análises bioquímicas foram realizadas pelo método enzimático colorimétrico utilizando o kit comercial Laborlab para análise da aspartato aminotransferase (AST). Para as demais análises, foram utilizados kits comerciais Bioclin.

O consumo de matéria seca (CMS) foi avaliado do $30^{\circ}$ ao $34^{\circ}$ dia do período experimental, e as amostras dos alimentos fornecidos aos equinos foram coletadas diariamente e armazenadas a $-18^{\circ} \mathrm{C}$. A produção fecal e as características das fezes dos equinos foram avaliadas no $33^{\circ}$ e no $34^{\circ}$ dia do período experimental. As fezes de cada equino foram coletadas imediatamente após a defecação e cuidadosamente sobrepostas em plástico azul e fotografadas.
As análises das características das fezes foram efetuadas segundo metodologia descrita por GONÇALVES et al. (2006), conforme os seguintes critérios: cor, consistência, presença ou ausência de corpos estranhos, feno e grãos mal digeridos. O critério cor foi categorizado em: normal (esverdeada), negra, avermelhada e amarelada. A consistência foi avaliada segundo os quesitos: normal, tipo ruminante (mais aquosa que as fezes consideradas normais), diarreicas e secas e pequenas. Após a avaliação, as fezes foram pesadas, e uma alíquota foi retirada e armazenada a $18^{\circ} \mathrm{C}$.

Ao término do ensaio, as amostras de alimentos e das fezes foram descongeladas à temperatura ambiente, homogeneizadas e retiradas amostras compostas individuais, que foram pré-secas em estufa de ventilação forçada a $55^{\circ} \mathrm{C}$, durante 72 horas, moídas a $2 \mathrm{~mm}$ e acondicionadas em sacos plásticos até a execução da análise de matéria seca, segundo procedimento descrito por SILVA\& QUEIROZ (2002).

Os resultados dos parâmetros hematológicos e bioquímicos, do consumo de matéria seca e da produção fecal foram submetidos à análise de variância, e as médias foram comparadas pelo teste de Student-Newman Keuls $(\mathrm{P}<0,05)$, utilizando o programa SAEG (2007). As características das fezes foram analisadas de forma descritiva segundo os critérios citados por GONÇALVES et al. (2006).

\section{RESULTADOS E DISCUSSÃO}

Os valores médios dos parâmetros hematológicos e bioquímicos dos equinos alimentados com dietas com diferentes níveis de inclusão de óleo de soja podem ser observados na tabela 2 . Não houve diferença no $\mathrm{VG}$ dos equinos alimentados com as dietas experimentais ( $\mathrm{P}>0,05)$, com valor médio de $32,8 \%$. MATTOS et al. (2006), avaliando equinos em atividade física de trote alongado durante duas horas e alimentados com dietas contendo 3,1 e 6,8\% de óleo de soja, observaram aumento significativo no VG dos equinos, com valores de 35,8 e $38,8 \%$, respectivamente, quando comparados com os animais alimentados com dieta de controle de $32,1 \%$. Os resultados contrastantes do VG podem estar relacionados à dieta, ao tipo de atividade física ou à raça dos animais, pois, em MATTOS et al. (2006), os equinos consumindo a dieta de controle apresentaram VG elevado em relação aos animais do presente trabalho. 
Tabela 2 - Valores médios da hematologia e da bioquímica sérica dos equinos alimentados com as dietas com inclusão de óleo de soja.

\begin{tabular}{|c|c|c|c|c|c|}
\hline \multirow{2}{*}{ Parâmetros } & \multicolumn{3}{|c|}{-------Níveis de inclusão de óleo de soja, \%------- } & \multirow{2}{*}{ DPR } & \multirow{2}{*}{ Valores de referência } \\
\hline & 0 & 8,5 & 19,5 & & \\
\hline \multicolumn{6}{|c|}{ Hematologia } \\
\hline Volume Globular (\%) & $30,2^{\mathrm{a}}$ & $35,4^{\mathrm{a}}$ & $32,6^{\mathrm{a}}$ & 8,8 & $38-42^{1}$ \\
\hline Eritrócitos $\left(10^{6} \mu \mathrm{L}^{-1}\right)$ & $6,0^{\mathrm{b}}$ & $8,0^{\mathrm{a}}$ & $7,5^{\mathrm{a}}$ & 13,2 & $7-11^{1}$ \\
\hline Hemoglobina $\left(\mathrm{g} \mathrm{dL}^{-1}\right)$ & $9,5^{\mathrm{b}}$ & $11,9^{\mathrm{a}}$ & $10,7^{\mathrm{ab}}$ & 8,3 & $11-17^{1}$ \\
\hline $\mathrm{VCM}(\mathrm{fL})$ & $51,1^{\mathrm{a}}$ & $44,5^{\mathrm{b}}$ & $43,7^{\mathrm{b}}$ & 6,8 & $42-47^{1}$ \\
\hline $\mathrm{CHCM}(\%)$ & $31,3^{\mathrm{a}}$ & $33,5^{\mathrm{a}}$ & $33,0^{\mathrm{a}}$ & 4,7 & $31-38,6^{2}$ \\
\hline Leucometria Global $\left(10^{3} \mu \mathrm{L}^{-1}\right)$ & $8,9^{\mathrm{a}}$ & $9,0^{\mathrm{a}}$ & $8,2^{\mathrm{a}}$ & 25,3 & $6-11^{1}$ \\
\hline Segmentados $\left(10^{3} \mu \mathrm{L}^{-1}\right)$ & $4,3^{\mathrm{a}}$ & $3,4^{\mathrm{a}}$ & $3,2^{\mathrm{a}}$ & 30,7 & $2,3-8,6^{3}$ \\
\hline Eosinófilos $\left(10^{3} \mu \mathrm{L}^{-1}\right)$ & $0,1^{\mathrm{a}}$ & $0,4^{\mathrm{a}}$ & $0,3^{\mathrm{a}}$ & 52,5 & $0-1^{1}$ \\
\hline Linfócitos $\left(10^{3} \mu \mathrm{L}^{-1}\right)$ & $4,0^{\mathrm{a}}$ & $4,6^{\mathrm{a}}$ & $4,4^{\mathrm{a}}$ & 30,6 & $2-5,5^{1}$ \\
\hline Monócitos $\left(10^{3} \mu \mathrm{L}^{-1}\right)$ & $0,4^{\mathrm{a}}$ & $0,5^{\mathrm{a}}$ & $0,4^{\mathrm{a}}$ & 65,1 & $0,2-0,8^{1}$ \\
\hline Sólidos Totais $\left(\mathrm{g} \mathrm{dL}^{-1}\right)$ & $8,2^{\mathrm{a}}$ & $8,1^{\mathrm{a}}$ & $8,4^{\mathrm{a}}$ & 4,6 & $5,5-7,5^{1}$ \\
\hline Fibrinogênio $\left(\mathrm{mg} \mathrm{dL}^{-1}\right)$ & 225,0 & 220,0 & 280,0 & - & $<400,0^{1}$ \\
\hline \multicolumn{6}{|c|}{ Bioquímica Sérica } \\
\hline Glicose $\left(\mathrm{mg} \mathrm{dL}^{-1}\right)$ & $82,0^{\mathrm{a}}$ & $87,4^{\mathrm{a}}$ & $88,8^{\mathrm{a}}$ & 5,9 & $70-140^{1}$ \\
\hline Triglicerídios $\left(\mathrm{mg} \mathrm{dL}^{-1}\right)$ & $39,5^{\mathrm{b}}$ & $46,2^{\mathrm{b}}$ & $57,0^{\mathrm{a}}$ & 15,0 & $40-44^{4}$ \\
\hline Colesterol $\left(\mathrm{mg} \mathrm{dL}^{-1}\right)$ & $105,2^{\mathrm{a}}$ & $130,2^{\mathrm{a}}$ & $132,8^{\mathrm{a}}$ & 16,6 & $75-150^{3}$ \\
\hline Uréia $\left(\mathrm{mg} \mathrm{dL}^{-1}\right)$ & $30,0^{\mathrm{a}}$ & $36,6^{\mathrm{a}}$ & $36,0^{\mathrm{a}}$ & 11,5 & $24-48^{1}$ \\
\hline Creatinina $\left(\mathrm{mg} \mathrm{dL}^{-1}\right)$ & $1,40^{\mathrm{a}}$ & $1,42^{\mathrm{a}}$ & $1,28^{\mathrm{a}}$ & 17,9 & $1,1-1,8^{1}$ \\
\hline$\gamma$-Glutamil Transferase $\left(\mathrm{U} \mathrm{L}^{-1}\right)$ & $19,7^{\mathrm{a}}$ & $15,8^{\mathrm{a}}$ & $19,2^{\mathrm{a}}$ & 37,3 & $10-40^{1}$ \\
\hline Aspartato Aminotransferase $\left(\mathrm{U} \mathrm{L}^{-1}\right)$ & $350,0^{\mathrm{a}}$ & $334,0^{\mathrm{a}}$ & $306,0^{\mathrm{a}}$ & 21,2 & $150-400^{1}$ \\
\hline Creatina Kinase $\left(\mathrm{U} \mathrm{L}^{-1}\right)$ & $108,8^{\mathrm{a}}$ & $101,4^{\mathrm{a}}$ & $101,4^{\mathrm{a}}$ & 15,0 & $100-300^{1}$ \\
\hline
\end{tabular}

DPR - Desvio padrão relativo

${ }^{1}$ ROSE \& HODGSON (1994); ${ }^{2}$ CARLSON (1993); ${ }^{3}$ ROBINSON (1997); ${ }^{4}$ HARVEY et al. (1997).

Médias nas linhas, seguidas por letras diferentes diferem pelo SNK $(\mathrm{P}<0,05)$.

VCM = Volume Corpuscular Médio.

$\mathrm{CHCM}=$ Concentração de Hemoglobina Corpuscular Média.

A inclusão de óleo de soja proporcionou acréscimo no número e redução no tamanho de eritrócitos $(\mathrm{VCM})(\mathrm{P}<0,05)$. Além disso, houve aumento na concentração de hemoglobina $(\mathrm{P}<0,05)$, sem haver alterações na $\mathrm{CHCM}$, que se deve ao maior número de eritrócitos, não alterando a concentração de hemoglobina intracelular. $\mathrm{O}$ aumento do número de eritrócitos nos equinos consumindo as dietas hiperlipidêmicas não pode ser evidenciado no VG devido às alterações observadas no VCM. Houve aumento compensatório do tamanho das hemácias nos equinos do grupo de controle, interferindo na quantidade de eritrócitos nos animais desse grupo e não alterando o VG. Essas alterações nos parâmetros supracitados sugerem uma melhora nos índices hematológicos dos equinos consumindo as dietas hiperlipidêmicas.

Em equinos da raça Brasileiro de Hipismo, LACERDA et al. (2006) observaram valores de VG de $33,5 \%$; eritrócitos de 7,84 x $10^{6} \mu \mathrm{L}^{-1}$; e hemoglobina de $11,3 \mathrm{~g} \mathrm{dL}^{-1}$ e citam que o fator racial e o tipo de atividade esportiva devem ser considerados nas interpretações dos parâmetros sanguíneos em equinos. Os valores supracitados são similares aos observados no presente trabalho, sugerindo que podem ser explicados pela semelhança do plantel de equinos utilizados. No entanto, RIBEIRO et al. (2008) observaram, em equinos da raça Pantaneira, castrados e em atividade esportiva, valores de $\mathrm{VG}$ e hemoglobinas de $32 \%$ e $11,9 \mathrm{~g} \mathrm{dL}^{-1}$, respectivamente, e valor do VCM de 53,0fL. A literatura corrente (ROSE \& HODGSON, 1994) cita maiores valores para VG e hemoglobina e menores para VCM, porém são utilizados como referência os equinos da raça Puro Sangue Inglês em atividade física de Turfe.

Não houve efeito das dietas na concentração sanguínea de sólidos totais $(\mathrm{P}>0,05)$. Entretanto, os valores estão até $0,9 \mathrm{~g} \mathrm{dL}^{-1}$ acima dos valores de citados por ROSE \& HODGSON (1994), com valor médio de $8,23 \mathrm{~g} \mathrm{dL}^{-1}$. Os valores de $\mathrm{VG}$ e sólidos totais observados neste trabalho indicam que não houve desidratação nos equinos, algo que seria esperado, pois os equinos estavam em repouso. 
Os valores de fibrinogênio e de leucometria global e específica não diferiram $(\mathrm{P}>0,05)$ entre os equinos consumindo as diferentes dietas experimentais, permanecendo dentro dos valores de normalidade relatados por ROSE \& HODGSON (1994) e ROBINSON (1997), evidenciando que os equinos estavam em estado hígido. No entanto, deve-se ressaltar que KINGSTON (2008) relata a ocorrência de diferenças na leucometria, em função da intensidade e duração do exercício nos equinos

A concentração plasmática de glicose nos equinos não foi influenciada pelas dietas $(\mathrm{P}>0,05)$, com valor médio de $86,06 \mathrm{mg} \mathrm{dL}^{-1}$, dentro da faixa dos valores de referência. DUREN et al. (1987), MANZANO et al. (1995), MARQUEZE et al. (2001), SLOET Van OLDRUITENBORGH-OOSTEBAAN et al. (2002) e MATTOS et al. (2006) também não observaram influência nos níveis plasmáticos de glicose em função do consumo de dietas hiperlipidêmicas em equinos. No entanto, HARKING et al. (1992) observaram aumento significativo nos níveis plasmáticos de glicose em equinos consumindo dietas com inclusão de óleo de milho equivalente a $10 \%$ da energia digestível. Esses resultados podem ser devidos à quantidade e à forma de fornecimento do óleo de soja e, principalmente, ao tempo de consumo das dietas. No presente trabalho, houve uma tendência de elevação da concentração de glicose nos equinos consumindo as dietas hiperlipidêmicas. No entanto, deve-se considerar que a variação observada nos artigos citados pode ser explicada pelo tempo de consumo e pela concentração de carboidratos hidrolisáveis nas dietas.

Os equinos consumindo dieta com 19,5\% de inclusão de óleo de soja apresentaram aumento $(\mathrm{P}<0,05)$ na concentração sérica de triglicerídios, em comparação com os animais consumindo as outras dietas. Nos equinos consumindo a dieta com $8,5 \%$ de inclusão de óleo de soja, houve aumento na concentração de triglicerídios, porém não significativo, de $6,7 \mathrm{mg} \mathrm{dL}^{-1}$, em relação aos equinos consumindo a dieta de controle. Os valores séricos de triglicerídios nos equinos consumindo as dietas hiperlipidêmicas estão acima dos valores de referência citados por HARVEY(1997).

O aumento de triglicerídios séricos é interessante em equinos atletas, pois a utilização metabólica dos triglicerídios retarda a utilização da glicose como fonte de energia no tecido muscular pela via anaeróbica, o que resulta em menor produção de lactato (PAGAN, 2001). No entanto, HARKING et al. (1992) não observaram alterações nas concentrações séricas de triglicerídios de equinos alimentados com dieta com inclusão de óleo de milho equivalente a $10 \%$ da energia digestível. E, ao contrário do observado no presente estudo, SLOET Van OLDRUITENBORGHOOSTEBAAN et al. (2002) observaram decréscimo significativo, de $5 \mathrm{mg} \mathrm{dL}^{-1}$, nas concentrações séricas de triglicerídios em equinos consumindo dieta com $11,8 \%$ de inclusão de óleo de soja. A redução nos níveis séricos dos triglicerídios pode ser relacionada à atividade física em testes de esforço submáximo, em esteira de alta velocidade.

Houve aumento, porém não significativo $(\mathrm{P}>0,05)$ nos níveis séricos de colesterol, nos equinos consumindo as dietas hiperlipidêmicas, de $26,3 \mathrm{mg} \mathrm{dL}^{-1}$ na média, em relação à dieta de controle, permanecendo dentro dos valores de referência citados por CARLSON (1993). Os valores de colesterol sérico nos equinos do presente trabalho podem ser devido ao tempo de consumo das dietas ou ao manejo alimentar utilizado, pois BOWMAN et al. (1977), utilizando dietas com 0, 5 , 10 e $20 \%$ de óleo de milho, MANZANO et al. (1995), fornecendo dietas com $0,5 \%$ de óleo de soja e 5,5\% de gordura animal e SLOET Van OLDRUITENBORGHOOSTEBAAN et al. (2002), utilizando dietas com 1,5 e $11,8 \%$ de óleo de soja verificaram aumento significativo na concentração de colesterol nos equinos consumindo as dietas hiperlipidêmicas.

A concentração sérica de ureia não foi alterada pelas dietas fornecidas aos equinos $(\mathrm{P}>0,05)$, podendo ponderar que as dietas utilizadas no presente trabalho estavam balanceadas, pois, segundo ROSE \& HODGSON (1994), o aumento na concentração plasmática de ureia em equinos em repouso, provavelmente, é devido ao aumento na proteína dietética, pois a ureia sanguínea é um derivado residual do metabolismo protéico.

Não houve diferença nas concentrações séricas de creatinina, GGT, AST e CK entre os equinos consumindo as dietas experimentais $(\mathrm{P}>0,05)$, permanecendo dentro da faixa de valores citados por HODGSON \& ROSE (1994). Os valores observados acima se devem ao fato de os equinos estarem em repouso por quatro dias consecutivos, não sendo influenciados pelo exercício físico. Além disso, podese inferir que a inclusão de óleo de soja nas dietas não afetou a saúde dos equinos, pois esses parâmetros são indicativos da função renal e do metabolismo hepático.

Os resultados do consumo de matéria seca (CMS), da produção fecal e das características das fezes dos equinos estão apresentados na tabela 3 . A inclusão de óleo de soja aumentou a densidade energética das dietas e reduziu o CMS, de 1,9 e $2,8 \mathrm{~kg}$, nas dietas com 8,5 e $19,5 \%$ de inclusão de óleo de soja, respectivamente, quando comparadas com a dieta de 
Tabela 3 - Consumo dietético, excreção fecal e características das fezes de equinos consumindo dietas com inclusão de óleo de soja.

\begin{tabular}{|c|c|c|c|c|}
\hline \multirow{2}{*}{ Item } & \multicolumn{4}{|c|}{ - } \\
\hline & 0 & 8,5 & 19,5 & DPR \\
\hline Consumo de matéria seca (kg MS dia ${ }^{-1}$ ) & $9,0^{\mathrm{a}} \pm 0,5$ & $7,1^{\mathrm{b}} \pm 0,8$ & $6,2^{\mathrm{c}} \pm 0,3$ & 7,4 \\
\hline Consumo de matéria seca $(\% \mathrm{PV})$ & $1,88^{\mathrm{a}} \pm 0,2$ & $1,55^{\mathrm{b}} \pm 0,1$ & $1,26^{\mathrm{c}} \pm 0,1$ & 9,8 \\
\hline Produção fecal (kg MS dia $\left.{ }^{-1}\right)$ & $3,4^{\mathrm{a}} \pm 0,6$ & $2,7^{\mathrm{b}} \pm 0,4$ & $2,3^{\mathrm{b}} \pm 0,5$ & 17,2 \\
\hline Produção fecal em matéria seca (\% PV) & $0,71^{\mathrm{a}} \pm 0,2$ & $0,58^{\mathrm{ab}} \pm 0,1$ & $0,47^{\mathrm{b}} \pm 0,1$ & 19,6 \\
\hline Produção fecal em matéria natural $\left(\mathrm{kg} \mathrm{dia}^{-1}\right)$ & $13,3^{\mathrm{a}} \pm 3,8$ & $9,3^{\mathrm{a}} \pm 1,8$ & $8,6^{\mathrm{a}} \pm 2,0$ & 19,6 \\
\hline Teor de água nas fezes $(\%)$ & $72,5^{\mathrm{a}} \pm 3,0$ & $69,9^{\mathrm{a}} \pm 2,1$ & $71,7^{\mathrm{a}} \pm 1,4$ & 3,1 \\
\hline
\end{tabular}

DPR - Desvio padrão relativo.

Médias nas linhas seguidas por letras diferentes diferem pelo SNK $(\mathrm{P}<0,05)$.

controle $(\mathrm{P}<0,05)$. A redução no CMS e, concomitantemente de carboidratos rapidamente fermentáveis contidos na ração concentrada, é benéfica aos equinos, pois reduz os riscos dos distúrbios gastrintestinais, como foi observado neste estudo, em que nenhum animal apresentou alterações do trato digestório. A redução no CMS proporcionou redução da produção fecal nos equinos consumindo as dietas com 8,5 e 19,5\% de óleo de soja, de 0,7 e 1,1 kg MS, respectivamente, em relação à dieta de controle $(\mathrm{P}>0,05)$. E, consequentemente, promoveu redução $(\mathrm{P}>0,05)$ nos valores médios diários da produção de matéria seca fecal em relação ao peso corporal dos equinos, de 18 e 34 pontos percentuais nas dietas com 8,5 e $19,5 \%$ de óleo de soja, respectivamente, quando comparadas com a dieta sem inclusão de óleo de soja.

A inclusão de 8,5 e 19,5\% de óleo de soja nas dietas dos equinos não influenciou a produção fecal diária na base da matéria natural, o teor de água e as características das fezes $(\mathrm{P}>0,05)$, com valores médios de $2,18 \%$ do peso vivo (PV) e $71,4 \%$ de umidade. Esses resultados são semelhantes ao observados na literatura, em equinos sadios e com dieta variada, com produção fecal diária de 1 a 3\% do PV, na matéria natural, e 75\% de umidade (MEYER, 1995).

A consistência das fezes foi considerada normal, sem aparência ressecada ou aquosa, com exceção de apenas um equino, que, consumindo a dieta de controle, produziu fezes com consistência mais aquosas que as demais. Esse animal possuía o vício de coprofagia, o que provavelmente influenciou a fisiologia digestiva e alterou a consistência fecal. Não foi observada a presença de corpos estranhos nas fezes, porém observou-se a presença de fenos e grãos mal digeridos, com maior frequência de grãos de aveia.

A inclusão do óleo de soja nos níveis utilizados neste estudo e o fornecimento de óleo de forma gradual para adaptação do trato digestivo não resultaram na ocorrência de diarreias ou cólicas, corroborando DUREN et al. (1987), os quais não observaram distúrbios gástricos e alterações nas características das fezes de equinos consumindo dietas com até $20 \%$ de inclusão de óleo de milho.

A ausência de alterações negativas nos parâmetros hematológicos e bioquímicos e das características das fezes indica que a inclusão de óleo de soja nas dietas de equinos pode ser utilizada para promover redução no CMS, propiciando a redução no consumo de carboidratos rapidamente fermentáveis e o menor peso do trato digestório durante os exercícios físicos que podem melhorar o desempenho atlético dos equinos.

\section{CONCLUSÕES}

A utilização de dietas hiperlipidêmicas para equinos com inclusão de óleo de soja melhorou os índices hematológicos, com aumento do número de eritrócitos e da concentração de hemoglobina. Os equinos alimentados com a dieta com 19,5\% de inclusão de óleo de soja apresentaram maiores níveis de triglicerídios séricos.

A utilização de dietas hiperlipidêmicas para equinos com inclusão de óleo de soja possibilita a redução do consumo dietético sem a ocorrência de diarreias ou alteração das características das fezes

\section{FONTE DE AQUISIÇÃO}

a - RH Rodeio - Socil

\section{REFERÊNCIAS}

ALMEIDA, M.I.V. et al. Composição química e predição do valor nutritivo de dietas para equinos. Revista Brasileira de Zootecnia, v.28, n.6, p.1268-1278, 1999.

BOWMAN, V.A. et al. Digestion of fat by equine. In: EQUINE NUTRITION AND PHYSIOLOGY SOCIETY SYMPOSIUM, 5., 1977, St. Louis. Proceedings... St. Louis: ENPS, 1977. p.40.

Ciência Rural, v.39, n.9, dez, 2009. 
CARLSON, G.P. Coleta de amostra e interprestação dos exames laboratoriais. In: SMITH, B.P. Tratado de medicina interna de grandes animais. São Paulo: Manole, 1993. Cap.4, p.395-424.

COLES, E.H. Patologia clínica veterinária. 3.ed. São Paulo: Manole, 1984. 566p.

DUREN, S.E. et al. Effect of dietary fat on blood parameters in exercised Thoroughbred horses. In: EQUINE EXERCISE PHYSIOLOGY, 2., 1987, Cambridge. Proceedings... Cambridge: ICEEP, 1987. p.674-685.

FRAPE, D. Equine nutrition and feeding. 3.ed. Oxford: Blackwell, 2004. 650p

GONCALVES, S. et al. Using feces characteristics as a criterion for the diagnosis of colic in the horse: a clinical review of 207 cases. Revue de Médecine Vétérinaire, n.157, v.1, p.3-10, 2006 Disponível em: <http://revmedvet.com/2006/RMV157_3_10.pdf> Acesso em: 15 jun. 2008.

HARKING, J.D. et al. Effect of added fat on racing performance in thoroughbred horse. Journal of Equine Veterinary Science, v.12 p.123-129, 1992. Disponivel em: <http://www.sciencedirect.com/ science?_ob=ArticleURL\&_udi=B 75GX-4R1KX09P \& u s e r $=687350 \& \quad$ c over D a t e $=04 \% 2$ F $30 \%$

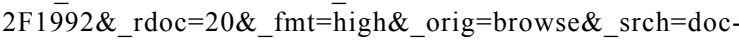
info $\% 23$ toc\%2313106\%231992\%23999879997\%23 $672556 \% 23$ FLP $\% 23$ display $\% 23$ Volume)\&_cdi=1310 $6 \&$ sort $=\mathrm{d} \&$ docanchor $=\& \quad \mathrm{ct}=31 \&$ acct $=\mathrm{C} 0000$ $37881 \&$ \&ersion $=1 \&$ urlVersion $=0$ \& userid $=687350 \& \mathrm{md} 5=\mathrm{ddc} 81 \mathrm{~b} 5$ 1a2300ebbab0b8bab6e1c135b>. Acesso em: 24 de agos. 2009. doi:10.1016/S0737-0806(06)81295-5.

HARVEY, J.W. The erythrocyte: physiology, metabolism and biochemical disorders. In: KANEKO, J.J. et al. Clinical biochemistry of domestic animals. 5.ed. San Diego: Academic, 1997. Cap.7, p.395-424

KINGSTON, J.K. Hematologic and serum biochemical responses to exercise and training. In: HINCHCLIFF, K.W. et al. Equine exercise physiology. Philadelphia: Saunders Elsevier. 2008. Cap.7, p.398-409.

LACERDA, L. et al. Hematologic and biochemical parameters in three high performance horse breeds from Southerm Brazil Archives of Veterinary Science, v.11, n.2, p.40-44, 2006. Disponível em: < http://ojs.c3sl.ufpr.br/ojs2/index.php/ veterinary/article/view/6783/4841>. Acesso em: 25 jul. 2008.

MANZANO, A. et al. Óleo de soja e gordura animal na alimentação de equinos. Revista Brasileira de Zootecnia, v.24, n.5, p.788-799, 1995.
MARQUEZE, A. et al. Aumento do nível de óleo em dietas isoenergéticas para cavalos submetidos a exercício. Ciência Rural, v.31, n.3, p.491-496, 2001. Disponível em: $<$ http:// www.scielo.br/scielo.php? script $=$ sci arttext\&pid $=\mathrm{S} 0103$ $84782001000300022 \& \operatorname{lng}=\mathrm{pt} \& \mathrm{nrm}=\mathrm{iso}>$. Acesso em: $25 \mathrm{jul}$. 2008. doi: 10.1590/S0103-84782001000300022

MATTOS, F. et al. Uso de óleo na dieta de equinos submetidos ao exercício. Revista Brasileira de Zootecnia, v.35, n.4, p.13731380, 2006. Disponível em: $<\mathrm{http} / /$ www.scielo.br/scielo.php?script $=$ sci arttext\&pid=S1516-35982006000500017\&lng=pt\&nrm=iso $>$. Acesso em: 17 jun. 2006. doi: 10.1590/S1516-35982006000500017.

MEYER, H. Alimentação de cavalos. São Paulo: Varela, 1995. 303p

NATIONAL RESEARCH COUNCIL - NRC. Nutrient requirements of horses. 5.ed. Rev. Washington, D.C.: National Academies, 1989. 100p.

NATIONAL RESEARCH COUNCIL - NRC. Nutrient requirements of horses. 6.ed. Rev. Washington, D.C.: National Academies, 2007. 360p

PAGAN, J.D. Recent developments in equine nutrition research. In: PAGAN, J.D. (Ed.) Advances in equine nutrition, 2. Kentucky: Kentucky Equine Research, 2001. p.251-258.

RIBEIRO, C.R. et al. Hematological profile of healthy Pantaneiro horses. Arquivo Brasileiro de Medicina Veterinária e Zootecnia, v.60, n.2, p. 492-495, 2008. Disponível em: $<$ http:/ /www.scielo.br/scielo.php? script $=$ sci arttext\&pid $=$ S0102$09352008000200033 \& \operatorname{lng}=$ pt\&nrm=iso $>$ Acesso em: 15 jul. 2008. doi: $10.1590 / \mathrm{S} 0102-09352008000200033$

ROBINSON, N.E. Current therapy in equine medicine, 4. Philadelphia: Saunders, 1997. 800p.

ROSE, R.J.; HODGSON, D,R. Hematology and biochemistry. In: HODGSON, D.R.; ROSE, R.J. The athletic horse: principles and practice of equine sports medicine. Philadelphia: Saunders, 1994. Cap.2, p.63-79.

SILVA, D.J.; QUEIROZ, A.C. Análise de alimentos - métodos químicos e biológicos. 3.ed. Viçosa: UFV, 2002. 235p

SLOET Van OLDRUITENBORGH-OOSTEBAAN, M. et al. Exercise-and metabolism-associated blood variables in Standardbreds fed either a low-or a high-fat diet. Equine Veterinary Journal, v.34, p.23-32, 2002.

UNIVERSIDADE FEDERAL DE VICCOSA. Sistema de análises estatísticas e genéticas, manual do usuário. Versão 9.1. Viçosa: UFV, 2007. 150p. 